\title{
Theoretical Predictions on the Effective Piezoelectric Coefficients of Wurtzite LaN compound from DFT and DFPT Calculations
}

\section{Z.A.A.R. Almaghbash}

Universite Dr Tahar Moulay de Saida

Arbouche Omar ( $\square$ arbouche_omar@yahoo.fr)

Universite Dr Tahar Moulay de Saida https://orcid.org/0000-0002-7406-5267
A. Cherifi
Universite Dr Tahar Moulay de Saida
B. Djellouli
Universite Dr Tahar Moulay de Saida

\section{Research Article}

Keywords: DFT, DFPT, PP-PW, piezoelectric properties, wurtzite LaN compound

Posted Date: October 28th, 2021

DOI: https://doi.org/10.21203/rs.3.rs-1010512/v1

License: (c) (i) This work is licensed under a Creative Commons Attribution 4.0 International License. Read Full License 


\title{
Theoretical predictions on the effective piezoelectric coefficients of wurtzite LaN compound from DFT and DFPT calculations
}

\author{
Z.A.A.R. Almaghbash ${ }^{1}$, O.Arbouche ${ }^{2,4}$, A.Cherifi ${ }^{1,4}$, and B. Djellouli ${ }^{3,4}$ \\ 1) Technology Laboratory of Communication, University of Saïda - Dr. Moulay Tahar, 20000 \\ Saïda, Algeria. \\ 2) Laboratory Physico-Chemistry of Advanced Materials, University of Sidi Bel-Abbes - \\ Djillali Liabes, 22000 Sidi BelAbbes, Algeria. \\ 3) Laboratoire de Modélisation Et Méthodes de Calcul LMMC, University of Saïda, Dr. \\ Moulay Tahar, 20000, Saïda, Algeria \\ 4) Department of Electronics, Faculty of Technology, University of Saïda, Dr. Moulay Tahar, \\ 20000, Saïda, Algeria
}

Corresponding author: arbouche_omar@yahoo.fr

zeyad.almaghbash@univ-saida.dz

\begin{abstract}
To study the structural, elastic, piezoelectric and dielectric properties of wurtzite (LaN), we have used the ab initio total-energy pseudo-potential plane-wave method (PP-PW) based on density functional theory (DFT) and density functional perturbation theory (DFPT), which performed with Generalized gradient approximation (GGA) as well as local density approximation (LDA). The calculations of piezoelectric constants by mean of linear-response calculations second derivative with respect to a strain and a modification of the electric field. The compound shows a large effective piezoelectric constant $\left(d_{33}\right)$ being in the order of 66.96, and $77.260 \mathrm{pc} / \mathrm{N}$ with (GGA), and (LDA) calculations, respectively. The dielectric properties and electromechanical coupling coefficients have been calculated. Finally, our piezoelectric findings indicate that the LaN compound has a high effective piezoelectric constant value $\left(d_{33}\right)$ which allows it to be in direct competitor of zinc oxide $\mathrm{ZnO}$ and aluminum nitride AIN. We hope that our findings provide guidelines for the experimental
\end{abstract}


realization and further research of high-performance materials appropriate for applications in different fields of study, such as biomedical engineering.

Keywords: DFT, DFPT; PP-PW; piezoelectric properties; wurtzite LaN compound

\section{Introduction:}

To date, due to their significant piezoelectric properties, zinc oxide $\mathrm{ZnO}$ and aluminum nitride AIN in the hexagonal structure have been materials widely used for medical ultrasound transducer applications. Moreover, in recent years, LaN compound has attracted growing attention in studies [1-4]. Recently, Ghezali, Mohamed, et al [5] conducted a study using the first principles of the LaN compound and showed the possibility of a crystal wurtzite structure B4 being present. The study also indicates that it is a stable semiconductor compound with a large indirect band gap of 0.999. In addition, Gazhulina, A. P [6], studied the electronic and optical properties of wurtzite LaN. The research indicated the stability of the compound and that it is among the semiconductors that can be used in various practical applications. Besides, the negative formation energy in the (OQMD) database was computed. [7] and checked in the frame of this study indicating stability against decomposition into constituent elements of LaN compound in hexagonal structure (P63mc) being in the order of $-1.426 \mathrm{eV} /$ atom implies the structural stability in these phase and suggest that they can be prepared and stabilized experimentally. In order to describe devices with extreme accuracy, there is a basic need to study materials and to know their physical properties that they include structural, elastic, piezoelectric and dielectric properties. For advanced systems, a greater understanding of structural properties offers a fundamental framework for the development of engineering materials. Calculated elastic constants provide information to measure mechanical properties such as rigidity, resilience, fragility, ductility. In addition, high-frequency piezoelectric materials have used in surface ultrasound processing to create transient acoustic waves. Among these phenomena is a study of rocket fuel burn accidents, shock and acceleration due to their response velocity close to a microsecond, i.e., rapid mechanical vibration. We focus on the theoretical investigation of the wurtzite AlN compound semiconductor in the present paper. After geometry optimization, we explain findings on structural, elastic, piezoelectric and dielectric properties, as programmed in the open source software package ABINIT [8] within the framework of DFT [9] and DFPT theories [10, 11], using both LDA [12] and GGA approximations [13] for functional exchange correlation. The rest of the paper is arranged on 
the basis of this. A brief overview of the methodology used and calculation description are given in Section 2. Section 3 presents and discusses the findings for structural, elastic, piezoelectric, dielectric and electromechanical coupling coefficients. In section 4, the conclusions are outlined.

\section{Theoretical methodology:}

Our calculations ab initio was carried out with the pseudo potential technique coupled with the base plane wave method (PW-PP) to solve the Kohn-Sham equation. This technique is based on the DFT density functional theory, where the exchange-correlation energy is treated with the two approximations: the local density (LDA; Local Density Approximation) On the basis of the Ceperley and Alder data [12] and Generalized Gradient Approximation (GGA) Using the Perdew-Burke-Ernzerhof scheme [13] pseudopotentials (Pseudo-dojo) generated by Van Setten, M. J, et al are used with LDA and GGA approximation [14]. ABINIT is a software for the calculation of the total energy, electronic structure and charge density of systems composed of electrons and nuclei with DFT, and DFPT, using pseudo-potentials and a plane wave base. ABINIT also provides options for simulation of molecular dynamics or dynamic matrix generation, effective Born charge, and elastic, dielectric and piezoelectric properties. We use a very high plane-wave energy cutoff of 60 Hartree to obtain high precision in the total energy estimation and ionic relaxation, and the convergence for optimum tolerance on the square residual wave function is set to $10^{-18}$. The k-point mesh used in our approximation is $10 \times 10 \times 10$ with relative energy converging to be $10^{-9}$, Using Monkhorst and Pack's typical k-point technique [15].The elastic tensor with relaxed atoms results from the combination of the second derivative for internal stress and the constants of the interatomic force; while the piezoelectric tensor with relaxed atoms takes into account the second derivatives for the internal stress, interatomic force constants, and Born effective charges. The following relation within the framework of DFPT used to define elastic constants:

$C_{i j}=\frac{1}{\Omega_{0}} \frac{\partial^{2} E}{\partial \eta_{i} \partial \eta_{j}}$

Where, $\Omega_{0}$ is the unit cell volume, $\eta_{i}$ and $\eta_{j}$ are the components of strain tensor [16] and Voigt's notation is used to denote $i, j=1,2, ., 6$. While the direct piezoelectric constants (e) relate the variation of the polarization $(\mathrm{P})$ at constant electric field $(\varepsilon)$ to stress undergone by solid as follows:

$$
P=e \eta
$$


The computational method consists of directly calculating the strain-induced polarization, since each piezoelectric stress coefficient could be defined as:

$$
e_{\alpha j}=\frac{\partial P_{\alpha}}{\eta_{j}}
$$

The piezoelectric constant Clamped - ion is defined as follows:

$$
e_{\alpha j}^{-}=\frac{\partial^{2} E}{\partial \sigma_{\alpha} \partial \eta_{j}}
$$

While, The internal-strain term of piezoelectric constant is determined as follows:

$$
e_{\alpha j}=\frac{1}{\Omega_{0}} Z_{m \alpha}\left(K^{-1}\right)_{m n} \Lambda_{n j}
$$

Hence through both equations we can obtain the proper piezoelectric constant:

$e_{\alpha j}=\frac{\partial^{2} E}{\partial \sigma_{\alpha} \partial \eta_{j}}+\frac{1}{\Omega_{0}} Z_{m \alpha}\left(K^{-1}\right)_{m n} \Lambda_{n j}$

With Z: the born effective charges.

In addition, the dielectric tensor $\epsilon_{\alpha \mathrm{j}}$ is defined as:

$$
\epsilon_{\alpha \mathrm{j}}=1+4 \Pi \frac{\partial P_{\alpha}}{\partial E_{j}}
$$

Finally, the tensor of the Born effective charges,

$$
Z_{\alpha}^{B} j(K)=\frac{V}{e} \partial \frac{P_{\alpha}}{\partial \mathrm{uj}(K)}
$$

All the formulas shown above are implemented in first-principles calculation packages ABINIT [8].

\section{Results and discussion}

\subsection{Structural and electronic properties}

The LaN compound belongs to the point group P63mc, with a Bravais lattice $(\mathrm{a}=4.115 \AA$ and $\mathrm{c}=5.908 \AA, \mathrm{u}=0.410$ ) [7]. The total energies determined versus unit cell volumes $\mathrm{E}(\mathrm{V})$ are shown in Figure 1; we fitted the determined energy with both LDA and GGA approximation versus volume points to the Birch-Murnaghan EOS [17]. Table 1 presents the determined lattice parameters a, c / a, c and $\mathrm{u}$, which also provides the theoretical data available for comparison. In view table 1, Our LDA-calculated in good agreement with the theorical [6]. In theoretical terms [7] a, and c are significantly underestimated. This is not the case when using GGA where our determined values are overestimated with respect to the theoretical for c constant [5]. By using the GGA method instead of the LDA one, the deviation tended to be larger. In terms of bulk modulus B and derivative of pressure B, our LDA results for wurtzite $\mathrm{LaN}$ are in good agreement with those of Gazhulina, A. P[6] for 
both B and B'. And although our GGA findings have been found to be less than theoretical [6]. our values for $\mathrm{c} / \mathrm{a}$ and $\mathrm{u}$ compared with the ideal structure $(\mathrm{c} / \mathrm{a}=8 / 3$ and $\mathrm{u}=3 / 8)$ [18], listed in table 1 for both LDA and GGA approximations. The structure of the energy bands shows that electronic energies are dependent on the $\mathrm{k}$ vector in the Brillouin zone in high symmetry directions. This structure provides a valuable mechanism for the qualitative study of the material's electronic properties. The electronic band structures with the LDA and GGA approximations of LaN compound along symmetry directions are displayed in Figure 2. We note that our compounds exhibit semiconductor behavior with a 0.97, $1.05 \mathrm{eV}$ indirect band gap with LDA and GGA approximations respectively, including the top of valance band (VB) at $\mathrm{K}$ and the bottom of the conduction band (CB) at $\Gamma$. Our results in both approximations show a similarity band gap with the theoretical with LDA and GGA [6].

\subsection{Elastic properties and related things}

The theory of elasticity deals with the behavior of materials which have the property of recovering their dimensions and their shapes when the forces which produce the deformations are removed. In the region near the equilibrium position of atoms, the energy of the solid can be considered as a quadratic function of the parameters of the solid. When stress is exerted on the crystal, it deforms, changing the parameters that describe it. These are the homogeneous deformations of the crystal. In the region close to equilibrium, the quadratic development of energy allows to express a linear relation between stress and strain: this is HOOK's law. This relation is defined thanks to the elastic constants. The elastic constants also make it possible to define the mechanical stability of the solid vis-a-vis the strains. Indeed, for the point of equilibrium to be a point of stable equilibrium, the quadratic form of the energy must be positive definite, which imposes conditions on the elastic constants. There are five separate elastic constants for a hexagonal system: $C_{11}, C_{12}, C_{13}, C_{33}$ and $C_{44}$. In the present work, with LDA, and GGA approximations, the elastic constants of the material are given in table 2.In view of table 2, Our results regarding elastic constants with LDA approximation were found to be larger than GGA approximation for all constants. We describe $\beta$ as the relative variation between axis $\mathrm{c}$ and axis a deformation, so $1 / \beta$ indicates linear compressibility anisotropy along axis $\mathrm{c}$ with respect to axis a, and a value of $1 / \beta$ equal to 1.0 indicates isotropic compressibility. It will be written in the form below [19]:

$\frac{1}{\beta}=\frac{C_{33}-C_{13}}{C_{11}+C_{12}-2 C_{13}}$ 
According to our results (see table 2), the anisotropies of linear compressibility are less than 1. However, we can note that for GGA, the anisotropy of linear compressibility is larger than that of LDA approximation.

For w-LaN, the Cij elastic constants satisfy mechanical stability conditions [20], namely $C_{44}>0, C_{11}^{2}>C_{12}^{2}$ and $\left(C_{11}+2 C_{12}\right) C_{33}>2 C_{12}^{2}$ indicates that w-LaN is stable in the above mentioned equations for both LDA and GGA approximations. Data of elastic constants allows us to determine shear modulus G, compressibility B, Young modulus E and Poisson's ratio v, which are important parameters in technical applications and offer a fundamental description of the behavior Mechanics of a material. If the independent elastic constants $C_{11}, C_{12}, C_{13}, C_{33}, C_{44}$ and $C_{66}=1 / 2\left(C_{11}-C_{12}\right)$ are all obtained, With Voigt and Reuss method, the polycrystalline module can be obtained. [21-23]. The elastic modulus $\left(B_{V}, B_{R}\right)$ and the shear module $\left(G_{V}, G_{R}\right)$ can be calculated as:

$$
\begin{gathered}
\left.B_{V}=\frac{1}{9}\left(2\left(C_{11}+C_{12}\right)+C_{33}+4 C_{13}\right)\right) \\
B_{R}=\frac{\left(C_{11}+C_{12}\right) C_{33}-2 C_{13}^{2}}{C_{11}+C_{12}+2 C_{33}-4 C_{13}}(12) \\
G_{V}=\frac{1}{30}\left(C_{11}+C_{12}+2 C_{33}-4 C_{13}+12 C_{44}+12 C_{66}\right),(13) \\
\left(C_{11}+C_{12}\right) C_{33}-2 C_{13}^{2}{ }^{2} C_{44} C_{66} \\
\left(C_{11}+C_{12}\right) C_{33}-2 C_{13}^{2}{ }^{2}\left(C_{44}+C_{66}\right) \\
3 B_{V} C_{44} C_{66}+ \\
G_{R}=\frac{5}{3}
\end{gathered}
$$

The elastic behavior of the material depends on the shear modulus $\mathrm{G}$ and bulk modulus B by using the approximations of Voigt $(\mathrm{V})$ and Reuss $(\mathrm{R})$ which are presented as follows: $B=$ $1 / 2\left(B_{R}+B_{V}\right)$ and shear modulus $G=1 / 2\left(G_{R}+G_{V}\right)$. From B and $\mathrm{G}$, Young modulus $\mathrm{E}$ is obtained as well: $E=9 B G /(3 B+G)$. All B, G, and E, with both LDA and GGA approximations, as shown in Table (3). The shear anisotropic parameter is essential for the understanding of elastic anisotropy to show the rate of anisotropy in atomic bonding on various crystallographic planes [24]. For hexagonal structure, shear anisotropies described as 
follows [25]: $\mathrm{A}=2 C_{44} /\left(C_{11}-C_{12}\right)$. Poisson's ratio is determined from the bulk $\mathrm{B}$ and the shear modulus $G$ as following $v=(3 B-2 G) / 2(3 B+G)$. According to the table 3 , The compressibility modulus B is higher in value compared to the shear modulus $\mathrm{G}$, therefore the material studied is more resistant to the change of volume (compression) than to the change of shape (shear). The determined A and $v$ for $\mathrm{w}-\mathrm{LaN}$ are all described in Table 3. Poisson's ratio $v$ is the transverse tension at extension or axial tension, when the sample is stretched. Its value is between -1 and 0.5 , the material is said to be covalent with an ionic character. The calculated values of the Poisson's ratio of LaN in wurtzite structure significantly higher than the critical value 0.25 , and therefore they have a large lateral expansion and they are unaffected by non-central forces. In addition, We note that GGA shear anisotropies (A) and Poisson 's ratio (v) smaller than calculated with LDA.

\subsection{Piezoelectric properties}

In this part, we will also discuss the possible piezoelectric effect of the LaN compound using the (DFT) and (DFPT) pseudo-potential plane wave (PP-PW) approach with both LDA and GGA functional energy exchange-correlation. In the case of insulators, which have their localized charges, the displacement of the atom following the deformation can cause a modification of the crystalline field (a macroscopic electric field) which can modify the polarization; some materials will therefore not be piezoelectric. The calculations of piezoelectric constants by mean of linear-response calculations second derivative with respect to a strain and a modification of the electric field. The properties of response to a macroscopic and static electric field can be calculated in the DFPT formalism. The binary LaN assumed in this work crystallizes in the structure of the wurtzite and has three separate piezoelectric tensor components, Specifically $e_{33}, e_{31}\left(=e_{32}\right)$, and $e_{15}\left(=e_{24}\right)$. Both the direct (e) and converse (d) relaxed and clamped-ion piezoelectric constants are included in Table 4. In the z-direction, w-LaN can easily be polarized, so the structure is strained in the same direction, for example. the largest one is the $e_{33}=x x x$ coefficient. We also note that the highest piezoelectric converse response occurs when the electrical field is applied along the zdirection leading to a strain in the same direction $\left(d_{33}\right)$, whereas the slight converse piezoelectric behaviour occurs along the yz $\left(d_{15}\right)$. As the strain is applied along yz, $n_{y z}\left(e_{15}\right)$, a minimum polarization takes place along yz. Interestingly enough, the major piezoelectric response occurs in the LaN crystal in the most rigid axis. In addition, theoretically, by decreasing the elastic constant $\left(C_{33}\right)$ and increasing the piezoelectric stress constant $\left(e_{33}\right)$, The 
piezoelectric constant $\left(d_{33}\right)$ can be increased. To check the functional dependence of the exchange-correlation on piezoelectric constants we performed the first principal calculation of

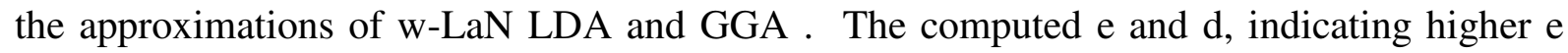
values than those determined by GGA obtained by LDA. The LDA-calculated d values appear to be higher than GGA's ones. To the best of our knowledge, there are no calculations regarding the piezoelectric properties and their comparison with our results. The findings of both LDA and GGA are of a qualitatively similar nature. Our piezoelectric findings indicate that the LaN compound has a large effective constant piezoelectric value $\left(d_{33}\right)$ that enables it to effectively compete with $\mathrm{ZnO}$ zinc oxide and $\mathrm{AIN}$ aluminum nitride.

\subsection{Dielectric properties}

The concept of solid dielectric behavior is an old one that is applicable to many electrondevice properties. [26]. The response to the perturbation of the electric field is used to deal with insulating material cases. It is possible, thanks to this response, to calculate the dielectric tensor $(\varepsilon(\infty))$ and the born effective charges $\left(Z^{B(k)}\right)$. The dielectric tensor can be divided into the contributions of ionic $\varepsilon_{i j}(0)$ and electronic $\varepsilon_{i j}(\infty)$. It consists of two separate components in the wurtzite structure, One that corresponds the direction parallel to the c-axis, $\varepsilon_{i}(\infty)=$ $\varepsilon_{z z}(\infty)$, The other is the hexagonal plane that is perpendicular to the c-axis, $\varepsilon_{j}=\varepsilon_{x x}(\infty)=$ $\varepsilon_{y y}(\infty)$. Our results concerning $\varepsilon_{i}(\infty)$ and $\varepsilon_{j}(\infty)$ for wurtzite LaN are found to be 7.06 and 7.02 with GGA and 7.12 and 7.44 for LDA approximation receptively. Our LDA results for $\varepsilon_{i}(\infty)$ and $\varepsilon_{j}(\infty)$ in good agreement with the theorical using LDA [6].and our GGA found to be larger than Theorical using GGA[6]. The average value $\varepsilon(\infty)=(1 / 3) \operatorname{Tr} \varepsilon(\infty)$ It has also been computed and found to be 7.01 and 7.21 for LDA, and GGA respectively. the total dielectric tensor constant are given as:

$\varepsilon_{i j}=\varepsilon_{i j}(0)+\varepsilon_{i j}(\infty)(15)$

The total dielectric constant of $\operatorname{LaN} \varepsilon_{11}(0)$, and $\varepsilon_{33}(0)$ calculated, and found to be 15.976, and 18.889 for LDA, 16.726, and 20.42 with GGA approximation. For the effective charges tensor, Because of the non-symmorphic space group $C_{6 v}^{4}$ with a screw axis along the c-axis and charging neutrality, one may consider only one Born tensor with two different components to be effective charges $Z_{T}^{B}=\left(Z^{B}\right)_{x x}=\left(Z^{B}\right)_{y y}$ and $Z_{I I}^{B}=\left(Z^{B}\right)_{z z}$. Furthermore, the average value $Z^{B}$ can be obtained as $Z^{B}=(1 / 3) \operatorname{Tr} Z^{B}$ Our calculations showed the values of $Z_{T}^{B}, Z_{I I}^{B}$, and $Z^{B}$ for wurtzite $\mathrm{LaN}$ with LDA are 3.54, 3.45 and 3.42, and 3.54, 3.52 and 3.5 for GGA approximations respectively . 


\subsection{Electromechanical coupling coefficients (ECF)}

Any piezoelectric material has an electromechanical coupling coefficient which translates its ability to transform an electrical quantity into a mechanical quantity and vice versa. This parameter takes into account both the elastic, dielectric and piezoelectric properties of the material. In the design of energy harvesting and sensing transducer applications, these coefficients are important criteria [27]. The electromechanical coupling coefficient depends on the direction of the applied electric field and the shape of the sample because the vibration modes change from one configuration or geometry to another. In various applications that require frequent the piezoelectric medium to work in linear length mode, the electromechanical coupling factor $K_{33}$ is important [28] such as transducer ultrasound and pressure sensors. It can be expressed in the following relationship:

$$
K_{33}^{2}=\frac{e_{33}^{2}}{\varepsilon_{33} C_{33}+e_{33}^{2}}
$$

Where $\varepsilon_{33}$ is the 33 components of the dielectric tensor. The high coupling factor of $K_{33}$ contributes to improved axial resolution, wider bandwidth and higher sensitivity transducers [29]. Cantilever-based actuators operate in the mode of transverse length (bending) for which the relevant coefficient of coupling is:

$$
K_{31}^{2}=\frac{e_{31}^{2}}{\varepsilon_{33} C_{11}+e_{31}^{2}}
$$

Our results concerning $K_{33}$ and $K_{31}$ for wurtzite LaN are calculated to be 0.47 , and 0.05 for LDA, 0.02 and 0.40 with GGA approximation respectively. Which is comparable with $\mathrm{ZnO}$ [30], AlN [31], BaTiO3 [32], and LiNbO3 [33].

\section{Conclusion}

In summary, the details of the structural, electronic, elastic, piezoelectric and dielectric properties of wurtzite LaN compound are being reported using calculations within density functional theory combined with density functional perturbation theory. We found that our compound exhibit semiconductor behavior with indirect band gap. Furthermore, our densityfunctional perturbation theory calculations reveal that the wurtzite $\mathrm{LaN}$ compounds materials have values of piezoelectric $\left(d_{33}\right)$ constant higher than that of the wurtzite $\mathrm{AlN}$ and $\mathrm{ZnO}$. The improvement in the piezoelectric value leads to a higher value in electromechanical coupling 
coefficients. A promising direction for further theoretical studies can be useful for piezoelectric thin film in the future.

\section{References}

[1] Vaitheeswaran, G., V. Kanchana, and M. Rajagopalan, Structural phase stability and superconductivity of LaN. Solid state communications 124, 97-102 (2002)

[2] Ciftci, Y. O., et al. "The first-principles study on the LaN." Materials Chemistry and Physics 108, 120-123 (2008)

[3] Hasegawa, Masashi, Ken Niwa, and Takehiko Yagi. "Synthesis of new La nitrides at high pressure and temperature." Solid state communications 141, 267-272 (2007)

[4] Li, Y. L., et al. "First principle study on the predicted phase transition of MN (M= Zr, La and Th)." Journal of Physics: Condensed Matter 31, 335402 (2019)

[5] Ghezali, Mohamed, et al. "Structural and electronic properties of LaN." Materials Chemistry and Physics 112, 774-778 (2008)

[6] Gazhulina, A. P. "A DFT investigation of 45 wurtzite (B4)-type compounds: Structural, electronic, linear and nonlinear optical properties." International Journal of Modern Physics B 34, 2050010 (2020)

[7] Saal, J. E., Kirklin, S., Aykol, M., Meredig, B., and Wolverton, “ The Open Quantum Materials Database (OQMD)", JOM 65, 1501-1509 (2013) https://doi.org10.1007/s11837013-0755-4

[8] Gonze, Xavier, et al. "First-principles computation of material properties: the ABINIT software project." Computational Materials Science 25, 478-492 (2002)

[9] Hohenberg, P., and W. Kohn. "Density functional theory (DFT)." Phys. Rev B864,136 (1964)

[10] Dal Corso, Andrea. "Density-functional perturbation theory with ultrasoft pseudopotentials." Physical Review B 64,235118 (2001)

[11] Gonze, Xavier, and Changyol Lee. "Dynamical matrices, Born effective charges, dielectric permittivity tensors, and interatomic force constants from density-functional perturbation theory." Physical Review B 55,10355 (1997) 
[12] Jollet, François, Marc Torrent, and Natalie Holzwarth. "Generation of Projector Augmented-Wave atomic data: A 71 element validated table in the XML format." Computer Physics Communications 1851246-1254 (2014)

[13] Perdew, John P., and Yue Wang. "Erratum: Accurate and simple analytic representation of the electron-gas correlation energy [Phys. Rev. B 45, 13244 (1992)]." Physical Review B 98,079904 (2018)

[14] Van Setten, M. J., et al. "The PseudoDojo: Training and grading a 85 element optimized norm-conserving pseudopotential table." Computer Physics Communications 226, 39-54 (2018)

[15] Monkhorst, Hendrik J., and James D. Pack. "Special points for Brillouin-zone integrations." Physical review B 13,5188 (1976)

[16] Doll, Klaus. "Analytical stress tensor and pressure calculations with the CRYSTAL code." Molecular Physics 108, 223-227(2010)

[17] Birch, Francis. "Finite elastic strain of cubic crystals." Physical review 71 ,809 (1947)

[18] Serrano, Jorge, et al. "Theoretical study of the relative stability of structural phases in group-III nitrides at high pressures." Physical Review B 62, 16612 (2000)

[19] Ravindran, P., et al. "Density functional theory for calculation of elastic properties of orthorhombic crystals: application to TiSi 2." Journal of Applied Physics 84,4891-4904 (1998)

[20] G.V. Sin'ko and N.A. Smirnow, Ab initio calculations of elastic constants and thermodynamic properties of bcc, fcc, and hcp Al crystals under pressure, J. Phys. Condens. Matter. 14, 6989-7005 (2002)

[21] D.M. Teter,Computational alchemy: The search for new superhard materials, MRS Bull. 23, 22-27 (1998)

[22] K. Tsubouchi and N. Mikoshiba, Zero-temperature-coefficient SAW devices on AlN epitaxial films, IEEE. Trans.Son. Ultrason. Su-32, 633-634 (1985)

[23] A. Reuss, Berechnung der Fliessgrenze von Mischkristallen auf Grund dPlastizitätsbedingung für Einkristalle, Math. Mech. 9, 49-58(1929)

[24] P. Ravindran, L. Fast, P.A. Korzhavyi, B. Johnnsson, J. Wills, and O. Eriksson, Density functional theory for calculation of elastic properties of orthorhombic crystals: Application to Ti Si2, J. Appl. Phys. 84 ,4891-4904 (1998)

[25] K.B. Panda and K.S. Ravi Chandran, Determination of elastic properties of titanium diboride (TiB2) from first principles using FLAPW implementation of the density functional theory, Comput. Mater. Sci. 35 ,134-150 (2006) 
[26] Sze, S. M. "Physics of Semiconductor Devices, Wiley, New York 1981." C. A. Mead, Analog VLSI and Neural Systems (Addison-Wesley, Reading, MA, (1989) pp 2959-2964.

[27] Wang, Qing-Ming, et al. "Electromechanical coupling and output efficiency of piezoelectric bending actuators." IEEE transactions on ultrasonics, ferroelectrics, and frequency control 46, 638-646 (1999)

[28] Kim, Moojoon, Jungsoon Kim, and Wenwu Cao. "Aspect ratio dependence of electromechanical coupling coefficient of piezoelectric resonators." Applied Physics Letters 87, 132901 (2005)

[29] Uchino, Kenji. "Piezoelectric ultrasonic motors: overview." Smart materials and structures 7, 273 (1998)

[30] Crisler, D. F., J. J. Cupal, and A. R. Moore. "Dielectric, piezoelectric, and electromechanical coupling constants of zinc oxide crystals." Proceedings of the IEEE 56, 225-226 (1968)

[31] Manna, Sukriti, et al. "Tuning the piezoelectric and mechanical properties of the AlN system via alloying with YN and BN." Journal of Applied Physics 122,105101 (2017)

[32] Hong, Kuang-Sheng, et al. "Direct water splitting through vibrating piezoelectric microfibers in water." The Journal of Physical Chemistry Letters 1, 997-10026 (2010)

[33] Turner, R. C., et al. Materials for high temperature acoustic and vibration sensors, A review. Applied acoustics 41,299-324 (1994) 
Figures
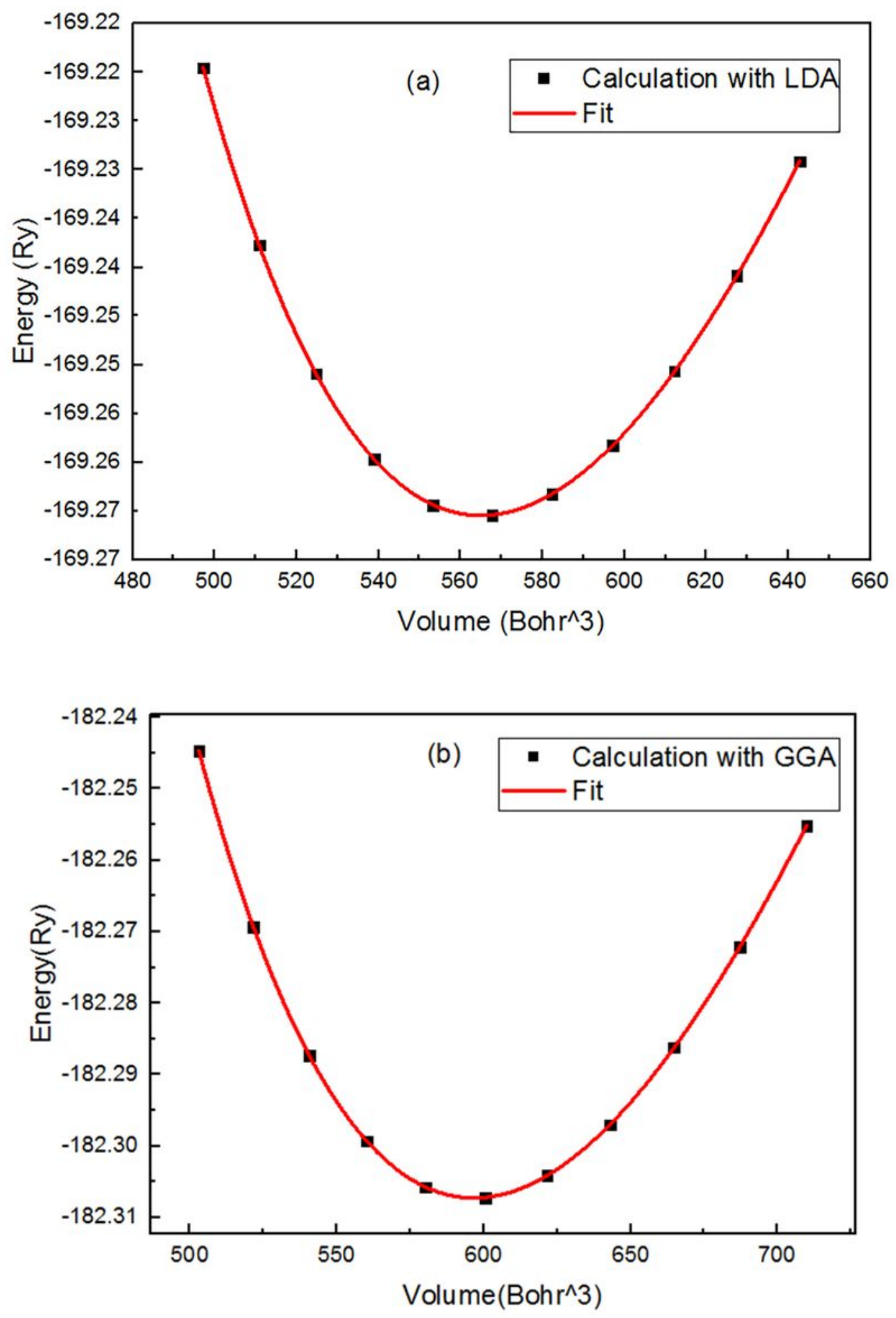

Figure 1

The total energies determined versus unit cell volumes $\mathrm{E}(\mathrm{V})$ 
(a) LaN-LDA

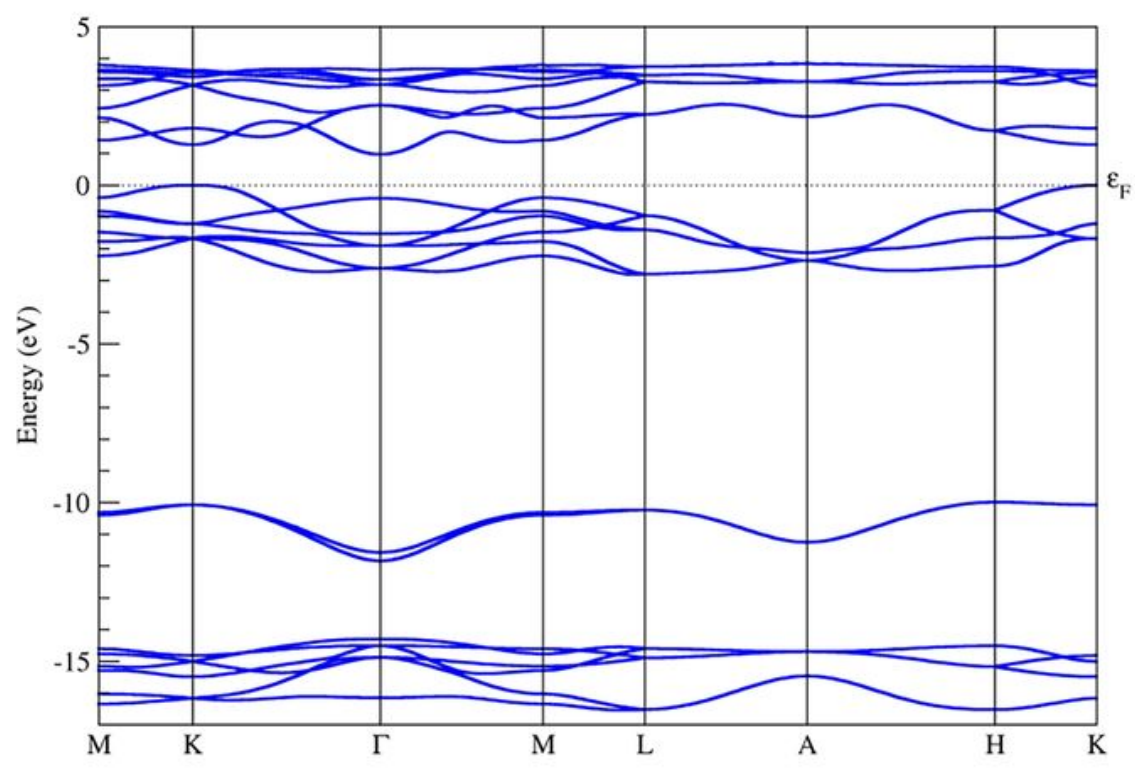

(b) LaN-GGA

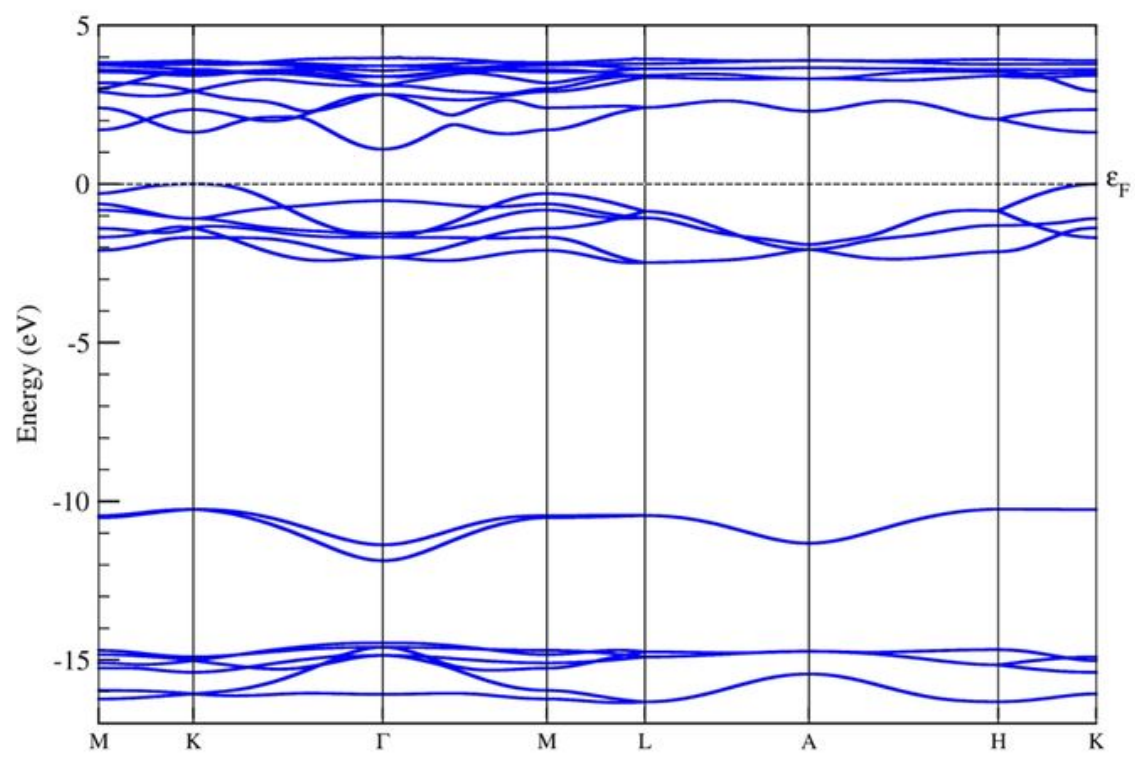

Figure 2

The electronic band structures with the LDA and GGA approximations of LaN compound along symmetry directions are displayed 